\title{
Schubert calculus and the homology of the Peterson variety
}

\author{
Erik Insko \\ Department of Mathematics \\ Florida Gulf Coast University \\ Fort Myers, FL 33965-6565, U.S.A. \\ einsko@fgcu.edu
}

Submitted: Dec 30, 2014; Accepted: May 5, 2015; Published: May 14, 2015

Mathematics Subject Classifications: 14F25, 14C17, 14L30, 14L35

\begin{abstract}
We use the tight correlation between the geometry of the Peterson variety and the combinatorics the symmetric group to prove that homology of the Peterson variety injects into the homology of the flag variety. Our proof counts the points of intersection between certain Schubert varieties in the full flag variety and the Peterson variety, and shows that these intersections are proper and transverse.
\end{abstract}

Keywords: Schubert calculus, intersection theory, Peterson variety

\section{Introduction}

The Peterson variety is a subvariety of the flag variety that has been the focus of much current research. In the early nineties Peterson announced that the quantum cohomology ring of any partial flag variety can be explicitly constructed as the coordinate ring of a certain subspace of the Peterson variety [13]. This spurred Kostant and Reitsch to study its geometry in connection to quantum cohomology $[10,15]$. Using the geometry of the Peterson variety, Reitsch also proved certain determinantal identities resembling classical results regarding Vandermonde determinants [14]. Brion and Carrell identified the $S^{1}$ equivariant cohomology of the Peterson variety with the coordinate ring of a particular affine curve [3]. In 2006, Tymoczko showed that a particular Schubert cell decomposition of the full flag variety, when intersected with the Peterson variety, paves the Peterson variety by affine cells $[16,17]$. More recently, Harada and Tymoczko used a modified GKM-theory to provide the first explicit computation of the $S^{1}$-equivariant cohomology of the Peterson varieties with generators and relations [7, Theorem 6.12, Corollary 6.17].

The following is a classic open problem in the Schubert calculus of the flag variety: 
Problem 1. Given any "reasonably nice" subvariety $Y \subset$ Flags $\left(\mathbb{C}^{n}\right)$, express the homology class of $Y$ as an integral linear combination of Schubert classes.

We consider the case where $Y$ is the Peterson variety. By analyzing the cellular structure of the Peterson variety and the group action of a one-dimensional torus on this variety, we reduce the computations in the intersection theory of the flag variety to a systematic combinatorial analysis of the elements of the symmetric group. In the process, we give a partial solution to Problem 1.

A precise statement of this result requires some further explanation and will appear as Theorem 17, but we provide a preview here:

Theorem 2. If we write every Peterson Schubert homology class as a linear combination of Schubert classes

$$
i_{*}\left(\left[\mathfrak{P e t} \cap X_{w_{J}}\right]\right)=\sum_{u} a_{u}\left[X_{u}\right]
$$

then each Peterson Schubert class $i_{*}\left(\left[\mathfrak{P e t} \cap X_{w_{J}}\right]\right)$ will have a certain coefficient $a_{v(J)}=1$, and the rest of the Peterson Schubert classes $i_{*}\left(\left[\mathfrak{P e t} \cap X_{w_{J^{\prime}}}\right]\right)$ will have $a_{v(J)}=0$.

It turns out that this result suffices to prove the main result of this paper.

Theorem 3. The push-forward $i_{*}: H_{*}(\mathfrak{P e t} ; \mathbb{Z}) \rightarrow H_{*}\left(\operatorname{Flags}\left(\mathbb{C}^{n}\right) ; \mathbb{Z}\right)$ induced by the natural inclusion map $i: \mathfrak{P e t} \hookrightarrow$ Flags $\left(\mathbb{C}^{n}\right)$ is injective.

Theorem 3 ensures that the Peterson variety is in fact one of the "reasonably nice" varieties described in Problem 1, and the push-forward $i_{*}$ being an injection is commonly necessary to obtain combinatorial constructions in equivariant cohomology, see e.g. [5]. Moreover, Theorem 3 gives geometric meaning to the combinatorial "Peterson Schubert classes" that Harada and Tymoczko described in their main results [7, Theorem 6.12, Corollary 6.17]; the combinatorial Peterson Schubert classes they described correspond to the fundamental classes of the Peterson Schubert varieties we describe in Section 3 and use in our intersection theory arguments. Most importantly, many of the intersection theory arguments laid out in Lie type A in this paper, can be generalized to all Lie types. Indeed, in a forthcoming paper, the author and Tymoczko use many of the techniques introduced in this paper to prove that the push-forward $i_{*}$ is an injection for the Peterson variety in any Lie type [9].

\subsection{Structure of this paper}

In Section 2 we set our conventions and recall some facts concerning the full flag variety. In Section 3 we examine the interplay between the geometry of the Peterson variety and the combinatorics of the symmetric group. In Section 4 we prove a series of lemmas leading up to the statement and proof of the main result. In Section 5 we conclude with an illustrative example and a list of open questions. 


\section{Flag varieties}

In this section we set our conventions concerning the full flag variety and its Schubert subvarieties, and we recall the fundamental facts concerning said varieties. General references for this material can be found in [1] or [6]. Let $V$ be an $n$-dimensional complex vector space. By a flag $V_{\bullet}$ in $V$ we mean a nested sequence of subspaces

$$
V_{\bullet}=V_{1} \subset V_{2} \subset \cdots \subset V_{n}
$$

such that $\operatorname{dim} \mathbb{C} V_{i}=i$ for all $1 \leqslant i \leqslant n$. The set of all flags $V_{\bullet}$ in the vector space $V$ forms a smooth complex projective variety known as the full flag variety, denoted Flags $\left(\mathbb{C}^{n}\right)$.

Fixing a basis $e_{1}, e_{2}, \ldots, e_{n}$ of $V$. Let $E_{\bullet}$ denote the standard flag spanned by the coordinate subspaces:

$$
E_{\bullet}=\left\langle e_{1}\right\rangle \subset\left\langle e_{1}, e_{2}\right\rangle \subset \cdots \subset\left\langle e_{1}, \ldots, e_{n}\right\rangle .
$$

Given any permutation $w \in S_{n}$ let $w\left(E_{\bullet}\right)$ denote the permutation flag:

$$
w\left(E_{\bullet}\right)=\left\langle e_{w(1)}\right\rangle \subset\left\langle e_{w(1)}, e_{w(2)}\right\rangle \subset \cdots \subset\left\langle e_{w(1)}, \ldots, e_{w(n)}\right\rangle .
$$

The group $G=G L_{n}(V)$ acts transitively on the set of all flags by left multiplication, and the Borel subgroup $B$ of upper triangular matrices is the stabilizer of this action. Hence the full flag variety Flags $\left(\mathbb{C}^{n}\right)$ may be identified with the quotient group $G / B$.

\subsection{Schubert cells and Schubert varieties}

The Bruhat decomposition theorem states that the group $G$ may be partitioned into double cosets:

$$
G=\coprod_{w \in S_{n}} B w B
$$

By restricting this decomposition to the quotient space $G / B$, the full flag variety Flags $\left(\mathbb{C}^{n}\right)$ may also be partitioned into cosets:

$$
\operatorname{Flags}\left(\mathbb{C}^{n}\right)=G / B=\coprod_{w \in S_{n}} B w B / B
$$

These cosets $B w B / B$ are isomorphic to affine cells, and the decomposition has the structure of a CW-complex. These cells are commonly refered to as Schubert cells. We denote the cell corresponding to a permutation $w$ in $S_{n}$ by $C_{w}$.

The closure of a Schubert cell is a subvariety of the full flag variety known as a Schubert variety, denoted $X_{w}$. The homology classes of the Schubert varieties $\left[X_{w}\right]$ form an additive $\mathbb{Z}$-module basis for the homology of the full flag variety $H_{*}\left(\operatorname{Flags}\left(\mathbb{C}^{n}\right)\right)$.

The opposite Schubert cell $C^{w}$ is the coset $B^{-} w B / B$ of the permutation matrix $w$ where $B^{-}$is Borel subgroup of lower triangular matrices. Similary, the opposite Schubert variety is defined to be the closure of the opposite Schubert cell $X^{w}=\overline{C^{w}}$. 


\subsection{Schubert classes}

The flag variety is a smooth projective variety, so it satisfies Poincaré duality. This means that there is a vector space isomorphism

$$
\rho: H_{2 k}\left(\operatorname{Flags}\left(\mathbb{C}^{n}\right) ; \mathbb{Z}\right) \rightarrow H^{n-2 k}\left(\operatorname{Flags}\left(\mathbb{C}^{n}\right) ; \mathbb{Z}\right)
$$

for each $k$. Hence for each Schubert variety $X_{w}$ there is a corresponding cohomology class $\rho\left(\left[X_{w}\right]\right)$, which we call a Schubert class.

The homology of a topological space does not usually have a ring structure. However the Poincaré duality map $\rho$ allows one to identify each graded piece of the cohomology ring $H^{2 n-2 k}\left(\operatorname{Flags}\left(\mathbb{C}^{n}\right) ; \mathbb{Z}\right)$ with the homology group $H_{2 k}\left(\operatorname{Flags}\left(\mathbb{C}^{n}\right) ; \mathbb{Z}\right)$.

Under this map the cup product in cohomology corresponds with the intersection of homology classes of subvarieties. In terms of Schubert classes, this means

$$
\rho\left[X_{w}\right] \cup \rho\left[X^{u}\right]=\rho\left[X_{w} \cap X^{u}\right] .
$$

Thus the Schubert classes form an additive $\mathbb{Z}$-basis that generates the cohomology ring of the flag variety. For a more comprehensive introduction to the intersection theory of Schubert varieties, consult Chapter 10 and Appendix B of Fulton's Young Tableau [6].

\subsection{Bruhat Order and Intersections of Schubert classes}

Let $s_{i}=(i, i+1)$ be a generator of the symmetric group $S_{n}$. It is well known that every permutation can be written as a product of these generators. A product of simple transpositions is called a reduced word when the product contains the minimal number of generators of any such representative of the permutation.

The length $\ell(w)$ of a permutation $w$ is the minimal number of simple transpositions in a reduced word decomposition of $w$. The Bruhat order on the permutation group specifies that a permutation $u$ is less than a permutation $w$ precisely when some (and hence every) reduced word of $u$ is a subword of some reduced word for $w$.

Geometry and combinatorics are intimately related in Schubert calculus. If the variety $X_{u}$ meets the variety $X^{v}$, then $\ell(v) \leqslant \ell(u)$. Equality holds if and only if $u$ equals $v$. The varieties $X_{u}$ and $X^{u}$ meet transversally at the point $u$. More concisely:

$$
\left[X_{u}\right] \cup\left[X^{v}\right]=\delta_{u}^{v}
$$

\section{The Peterson variety}

Let $N$ be a nilpotent linear operator on $V$ satisfying $N^{k} \neq 0$ for each $1 \leqslant k \leqslant n-2$ and $N^{n-1}=0$. Up to a change of basis for $V$ we may take $N$ to be the nilpotent matrix with 1 's along the super-diagonal.

Definition 4. The Peterson variety $\mathfrak{P e t}$ is the subvariety of the full flag variety defined by

$$
\mathfrak{P e t}=\left\{V_{\bullet} \in \operatorname{Flags}\left(\mathbb{C}^{n}\right) \mid N \cdot V_{i} \subset V_{i+1} \text { for all } 1 \leqslant i<n\right\}
$$


It has been extensively studied by Dale Peterson, who announced that the quantum cohomology ring of any partial flag variety $G / P$ can be identified with the coordinate ring of the intersection of the opposite Schubert cell $C^{w_{P}}$ with the Peterson variety. In other words, the geometry of the Peterson variety completely encodes the quantum cohomology of every partial flag variety. This result was published in type $A_{n-1}$ by [14]

Tymoczko showed that the intersections of the Schubert cells with the Peterson variety form a paving by affines of the Peterson variety [16]. This implies that the fundamental classes of the closures of these cells form a $\mathbb{Z}$-module basis for the homology of the Peterson variety [4, Examples 1.9.1 and 19.1.11(b)]. For this reason we make the following definition.

Definition 5. We define a Peterson Schubert cell to be the intersection of a Schubert cell $C_{w}$ with the Peterson variety, and a Peterson Schubert variety to be the intersection of a Schubert variety with the Peterson variety. We will denote these spaces $C_{w}^{\prime}$ and $X_{w}^{\prime}$ respectively.

\subsection{Geometry of the Peterson variety}

A fundamental tool when studying flag varieties is to analyze their group actions. Let $T$ denote the diagonal matrices in $\mathrm{GL}_{n}(V)$, and let $T$ act on Flags $\left(\mathbb{C}^{n}\right)$ by left multiplication. Schubert cells, and hence Schubert varieties, are invariant under this action. Moreover, the $T$-fixed point set of the flag variety $\operatorname{Flags}\left(\mathbb{C}^{n}\right)^{T}$ is the set of permutation flags, and the $T$-fixed point set of the flag variety restricts to the $T$-fixed point set of each Schubert subvariety

$$
X_{w}^{T}=X_{w} \cap \operatorname{Flags}\left(\mathbb{C}^{n}\right)^{T} .
$$

Sadly, the action of $T$ on the flag variety does not preserve the Peterson variety. However Harada and Tymoczko observed that there is a subtorus $S \subset T$ which acts on the Peterson variety and maintains many of the desirable properties of the $T$-action on Schubert varieties.

Proposition 6. [7, Facts 2.1-2.3] Let $S$ be the subtorus of $T$ consisting of diagonal matrices of the form $S=\left\{\left(s^{1}, s^{2}, \ldots, s^{n}\right) \mid s \in \mathbb{C}^{*}\right\} \subset T$. Then following statements hold:

1. The torus $S$ preserves the Peterson variety.

2. The $S$-fixed point set of the flag variety equals that of $T$

$$
\operatorname{Flags}\left(\mathbb{C}^{n}\right)^{S}=\operatorname{Flags}\left(\mathbb{C}^{n}\right)^{T}
$$

3. The S-fixed point set of the flag variety restricts to that of the Peterson variety

$$
\mathfrak{P e t}^{S}=\mathfrak{P e t} \cap \operatorname{Flags}\left(\mathbb{C}^{n}\right)^{S} .
$$

The Schubert cells are $S$-invariant since they are $T$-invariant, and it follows that the Peterson Schubert cells are also $S$-invariant. This fact leads us to the following lemma. 
Lemma 7. The Schubert cell $C_{w}$ intersects the Peterson variety if and only if the permutation flag $w\left(E_{\bullet}\right)$ lies in the Peterson variety.

The proof follows from the fact that $C_{w}$ and $\mathfrak{P e t}$ are $S$-invariant, and thus the intersection is a closed $S$-invariant subvariety of $C_{w}$. Hence the fixed point must be contained in this intersection. This lemma suggests that the cellular structure of the Peterson variety can be understood by studying the combinatorics of its fixed points. In the next subsection, we will give a nice characterization of the permutations correspond to cells in the Peterson variety.

\subsection{Combinatorics of Peterson variety}

Let $J \subset S_{n}$ denote the subgroup of the symmetric group $S_{n}$ generated by a set of simple transpositions $\left\{s_{i_{1}}, s_{i_{2}}, \ldots, s_{i_{|J|}}\right\}$, and let $w_{J}$ denote the longest word in $J$.

To identify which $S$-fixed points intersect the Peterson one simply checks which permutation flags $w\left(E_{\bullet}\right)$ satisfy $N\left\langle e_{w(i)}\right\rangle \subset\left\langle e_{w(i+1)}\right\rangle$ for all $1 \leqslant i<n$. This leads to the following description of the $S$-fixed points.

Lemma 8. [7] An S-fixed point $w\left(E_{\bullet}\right)$ is contained in the Peterson variety if and only if $w$ satisfies $w^{-1}(i) \leqslant w^{-1}(i+1)+1$ for all $1 \leqslant i<n$.

It follows immediately that we have the following standard, yet nonetheless remarkable, description of the $S$-fixed points.

Fact 9. The $S$-fixed points of the Peterson variety are precisely the permutation flags $w_{J}\left(E_{\bullet}\right)$ corresponding to the subgroups $J$ of $S_{n}$.

In light of this result, we will henceforth denote the $S$-fixed points of the Peterson variety by $w_{J}\left(E_{\bullet}\right)$ to emphasize their dependence on subgroups of $S_{n}$.

Example 10. The fixed points of $\mathfrak{P e t} \subset \mathcal{F} \ell_{4}(V)$ correspond to the following permutations written in one-line notation

$$
\text { 4321, 3214, 2143, 1432, 2134, 1324, 1243, } 1234 .
$$

The condition that $N V_{i} \subset V_{i+1}$ implies that each Peterson Schubert cell is blockdiagonal and that the nonzero entries of these blocks must repeat along each anti-diagonal. This leads to another remarkable relationship between the combinatorics of the $S$-fixed point set $\mathfrak{P e t}^{S}$ and the geometry of the Peterson variety.

Lemma 11. For each fixed point $w_{J}\left(E_{\bullet}\right)$ in $\mathfrak{P e t}^{S}$, the dimension of the cell $C_{w_{J}}^{\prime}$ is equal to the number of simple transpositions generating $J$.

Proof. This lemma is a corollary to Theorem 4.3 of Tymoczko's 2006 article Paving Hessenbergs by Affines [17, Theorem 4.3]. Tymoczko showed that the dimension of $C_{w_{J}}^{\prime}$ is equal to the number of positive simple roots that are mapped to negative simple roots by $w_{J}$. Each of these positive simple roots corresponds to a simple transposition $s_{i}$ generating $J$. Thus, the dimension of $C_{w_{J}}^{\prime}$ is the number of simple transpositions generating $J$. 
The following example illustrates the structure of the cells described in Lemma 11.

Example 12. The Peterson Schubert cells $C_{w_{J}}^{\prime}$ and $C_{w_{J^{\prime}}}^{\prime}$ corresponding to the subgroups $J$ and $J^{\prime}$ generated by $\left\{s_{2}, s_{3}, s_{4}\right\}$ and $\left\{s_{1}, s_{2}, s_{4}\right\}$ are depicted below:

$$
C_{w_{J}}^{\prime}=\left\{\left(\begin{array}{c|cccc}
1 & 0 & 0 & 0 & 0 \\
\hline 0 & a & b & c & 1 \\
0 & b & c & 1 & 0 \\
0 & c & 1 & 0 & 0 \\
0 & 1 & 0 & 0 & 0
\end{array}\right)\right\} \quad C_{w_{J^{\prime}}}^{\prime}=\left\{\left(\begin{array}{ccc|cc}
a & b & 1 & 0 & 0 \\
b & 1 & 0 & 0 & 0 \\
1 & 0 & 0 & 0 & 0 \\
\hline 0 & 0 & 0 & c & 1 \\
0 & 0 & 0 & 1 & 0
\end{array}\right)\right\}
$$

\section{The main result}

Since the Peterson variety is a closed subvariety of the full flag variety, the topological inclusion map $i: \mathfrak{P e t} \hookrightarrow$ Flags $\left(\mathbb{C}^{n}\right)$ induces a covariant map on homology

$$
i_{*}: H_{*}(\mathfrak{P e t}, \mathbb{Z}) \rightarrow H_{*}\left(\operatorname{Flags}\left(\mathbb{C}^{n}\right), \mathbb{Z}\right) .
$$

Let $\left[X_{w_{J}}^{\prime}\right]$ denote the homology class of a Peterson Schubert variety in $H_{*}(\mathfrak{P e t})$. The push-forward $i_{*}\left(\left[X_{w_{J}}^{\prime}\right]\right)$ is a homology class in $H_{*}\left(\operatorname{Flags}\left(\mathbb{C}^{n}\right)\right)$. Since the classes of Schubert varieties $\left[X_{w}\right]$ form a basis of $H_{*}\left(\right.$ Flags $\left.\left(\mathbb{C}^{n}\right)\right)$ as a $\mathbb{Z}$-module, we may express the pushforward of each Peterson Schubert homology class as an integral linear combination of the homology classes of the Schubert varieties, i.e.,

$$
i_{*}\left(\left[X_{w_{J}}^{\prime}\right]\right)=\sum_{u \in S_{n}} a_{u}\left[X_{u}\right]
$$

where $a_{u}$ are integers. Before proving Theorems 17 and 18 we will need the following three lemmas.

Let $J$ be a subgroup of $S_{n}$ generated by a set of simple transpositions $\left\{s_{i_{1}}, s_{i_{2}}, \ldots s_{i_{|J|}}\right\}$. Define the permutations $v(J)$ and $u(J)$ to be

$$
v(J)=s_{i_{1}} s_{i_{2}} \cdots s_{i_{|J|}} \quad \text { and } \quad u(J)=s_{i_{|J|}} s_{i_{|J|-1}} \cdots s_{i_{1}} .
$$

Henceforth, we state results in terms of $\left[X^{v(J)}\right]$, but the same statements hold for $\left[X^{u(J)}\right]$ as well.

Lemma 13. Let $\left[X_{w_{J}}^{\prime}\right]$ be a Peterson Schubert class in $H_{k}(\mathfrak{P e t})$. The Schubert variety $X^{v(J)}$ possesses the following properties:

1. The dimension of the flag variety is related to the dimensions of $X^{v(J)}$ and $X_{w_{J}}^{\prime}$ by

$$
\operatorname{dim} X^{v(J)}+\operatorname{dim} X_{w_{J}}^{\prime}=\operatorname{dim} \operatorname{Flags}\left(\mathbb{C}^{n}\right) .
$$

2. The varieties $X^{v(J)}$ and $X_{w_{J}}^{\prime}$ intersect in the point $w_{J}\left(E_{\bullet}\right)$,

$$
X^{v(J)} \cap X_{w_{J}}^{\prime}=w_{J}\left(E_{\bullet}\right) .
$$


Proof. It is a well-known fact that

$$
\operatorname{dim} X^{v(J)}=\operatorname{dim} \operatorname{Flags}\left(\mathbb{C}^{n}\right)-\ell(v(J)) .
$$

Lemma 11 says that $\operatorname{dim}\left(X_{w_{J}}^{\prime}\right)=\ell(v(J))$. This proves $(1)$.

Since $X_{w_{J}}^{\prime}$ and $X^{v(J)}$ are closed $S$-invariant subvarieties of the flag variety, their intersection is too. Any closed $S$-invariant subspace of dimension at least one contains at least two fixed points. However, we will show that there is only one fixed point in this intersection, and thus this must be the only point in the intersection.

The $S$-fixed points in $X_{w_{J}} \cap X^{v(J)}$ are those $w\left(E_{\bullet}\right)$ with $v(J) \leqslant w \leqslant w_{J}$ in Bruhat order $\left[1\right.$, Section 2.7]. Of these fixed points $w_{J}\left(E_{\bullet}\right)$ is the only one that corresponds to a subgroup, and this proves (2).

Lemma 13 proves that the varieties $X_{w_{J}}^{\prime}$ and $X^{v(J)}$ intersect properly at the point $w_{J}\left(E_{\bullet}\right)$. The following result shows that the intersection $X_{w_{J}}^{\prime} \cap X^{v\left(J^{\prime}\right)}$ for any two distinct subgroups $J, J^{\prime} \leqslant S_{n}$ with the same number of generators.

Lemma 14. Let $J$ and $J^{\prime}$ be two distinct subgroups of $S_{n}$ with the same number of number of simple transpositions as generators. Then the intersection $X_{w_{J}}^{\prime} \cap X^{v\left(J^{\prime}\right)}$ is empty.

Proof. The proof is similar to that of Lemma 13. The intersection $X_{w_{J}}^{\prime} \cap X^{v\left(J^{\prime}\right)}$ is a closed $S$-invariant subvariety. There are no permutations $w$ with $v\left(J^{\prime}\right) \leqslant w \leqslant w_{J}$ in the Bruhat order because $v\left(J^{\prime}\right)$ contains a simple transposition that is not in $w_{J}$. Hence $X_{w_{J}} \cap X^{v\left(J^{\prime}\right)}$ do not share any common $S$-fixed points. Thus the intersection is empty.

Lemma 15. The variety $X^{v(J)}$ intersects $X_{w_{J}}^{\prime}$ transversally at the point $w_{J}\left(E_{\bullet}\right)$.

Proof. The Peterson Schubert cell $C_{w_{J}}^{\prime}$ is a dense open neighborhood of $w_{J}\left(E_{\bullet}\right)$ in $X_{w_{J}}^{\prime}$. Every flag in this cell corresponds to a matrix that is block-diagonal with nonzero entries repeating along the anti-diagonals of each block. The neighborhood $N^{w}$ of $w\left(E_{\bullet}\right)$ in $X^{v}$ is described in Section 3.2 of Woo and Yong's Governing Singularities of Schubert varieties article [18, Section 3.2]. Following their construction, we see that an arbitrary flag in the neighborhood $N^{w_{J}}$ of $w_{J}\left(E_{\bullet}\right)$ in $X^{v(J)}$ corresponds to a matrix with with zeros across the top row of each diagonal block of $w_{J}$, nonzero free entries below the top row of each block, and nonzero free entries below each diagonal block.

Since an arbitrary matrix in $C_{w_{J}}^{\prime}$ is zero outside of the diagonal blocks, and a matrix in $N^{w_{J}}$ has zeros across the top row of each diagonal block, the only point of intersection of these two neighborhoods occurs when all of the free entries are zero in the matrix representatives of $N^{w_{J}}$ and $C_{w_{J}}^{\prime}$.

The only matrix in this intersection is the one corresponding to $w_{J}\left(E_{\bullet}\right)$. We can conclude that both neighborhoods are linear spaces which are isomorphic to their tangent spaces, and these cells intersect properly in the single fixed point. Thus the corresponding tangent spaces intersect $T_{w_{J}\left(E_{\bullet}\right)}\left(X^{v_{J}}\right) \cap T_{w_{J}\left(E_{\bullet}\right)}\left(X_{w_{J}}\right)=w_{J}\left(E_{\bullet}\right)$ in only one point. Since

$$
\operatorname{dim} T_{w_{J}\left(E_{\bullet}\right)}\left(X^{v_{J}}\right)+\operatorname{dim} T_{w_{J}\left(E_{\bullet}\right)}\left(X_{w_{J}}^{\prime}\right)=\operatorname{dim} T_{w_{J}\left(E_{\bullet}\right)}\left(F \operatorname{lags}\left(\mathbb{C}^{n}\right)\right)
$$

we have that $T_{w_{J}\left(E_{\bullet}\right)}\left(X^{v_{J}}\right) \oplus T_{w_{J}\left(E_{\bullet}\right)}\left(X_{w_{J}}^{\prime}\right)=T_{w_{J}\left(E_{\bullet}\right)}\left(\operatorname{Flags}\left(\mathbb{C}^{n}\right)\right)$. Thus the intersection is transverse. 
Example 16. Let $J=\left\{s_{1}, s_{3}, s_{4}\right\} \subset S_{5}$. The neighborhoods of $w_{J}\left(E_{\bullet}\right)$ in $X_{w_{J}}^{\prime}$ and $X^{v(J)}$ appear below:

$$
C_{w_{J}}^{\prime}=\left\{\left(\begin{array}{cc|ccc}
a & 1 & 0 & 0 & 0 \\
1 & 0 & 0 & 0 & 0 \\
\hline 0 & 0 & b & c & 1 \\
0 & 0 & c & 1 & 0 \\
0 & 0 & 1 & 0 & 0
\end{array}\right)\right\} \quad N^{w_{J}}\left\{\left(\begin{array}{cc|ccc}
0 & 1 & 0 & 0 & 0 \\
1 & 0 & 0 & 0 & 0 \\
\hline x & y & 0 & 0 & 1 \\
z & w & u & 1 & 0 \\
v & t & 1 & 0 & 0
\end{array}\right)\right\} .
$$

By examining these cells one can verify Lemmas 13 and 15. Indeed these cells are clearly linear spaces. The dimensions of these two cells add up to the dimension of the flag variety. The only point of intersection between $C_{w_{J}}^{\prime}$ and $N^{w_{J}}$ is where all of the free entries are zero. This is precisely the fixed point $w_{J}\left(E_{\bullet}\right)$.

Looking back at Example 12, one may note that the neighborhood $N^{w_{J}}$ of $w_{J}\left(E_{\bullet}\right)$ in $X^{v(J)}$ does not intersect either of the cells in that example. This is in accordance with Lemma 14.

We are now prepared to state our main results.

Theorem 17. If we write the push-forwards of the Peterson Schubert classes in terms of Schubert classes

$$
i_{*}\left(\left[X_{w_{J}}^{\prime}\right]\right)=\sum_{u \in S_{n}} a_{u}\left[X_{u}\right]
$$

then for every Peterson Schubert class $\left[X_{w_{J}}^{\prime}\right]$ the coefficient of $a_{v(J)}$ is 1 , and for every other Peterson Schubert class $\left[X_{w_{J^{\prime}}}^{\prime}\right]$ the coefficient of $a_{v(J)}=0$.

Proof. Let $X_{w_{J}}^{\prime}$ be a Peterson Schubert variety. The expression for $i_{*}\left(\left[X_{w_{J}}^{\prime}\right]\right)$ in terms of Schubert classes is

$$
i_{*}\left(\left[X_{w_{J}}^{\prime}\right]\right)=\sum_{u \in S_{n}} a_{u}\left[X_{u}\right]
$$

where the sum runs over all $u \in S_{n}$ such that $\ell(u)$ is equal to the number of simple transpositions generating the subgroup $J \leqslant S_{n}$. Lemmas 13 and 15 prove that $X_{w_{J}}^{\prime}$ and $X^{v(J)}$ intersect properly and transversely in a single point. This implies that $\left[X_{w_{J}}^{\prime}\right] \cup$ $\left[X^{v(J)}\right]=1$, and thus the coefficient $a_{v(J)}$ is 1 in the expansion of $i_{*}\left(\left[X_{w_{J}}^{\prime}\right]\right)$.

Lemma 14 proves $\left[X_{w_{J^{\prime}}}^{\prime}\right] \cup\left[X^{v(J)}\right]=0$ for $J^{\prime} \neq J$. This implies that the coefficient $a_{v(J)}=0$ in the expansion of all other generators $i_{*}\left(\left[X_{w_{J^{\prime}}}^{\prime}\right]\right)=\sum_{u \in S_{n}} a_{u}\left[X_{u}\right]$.

Theorem 18. The push-forward $i_{*}: H_{*}(\mathfrak{P e t} ; \mathbb{Z}) \rightarrow H_{*}\left(\right.$ Flags $\left.\left(\mathbb{C}^{n}\right) ; \mathbb{Z}\right)$ induced by the natural inclusion map $i: \mathfrak{P e t} \hookrightarrow$ Flags $\left(\mathbb{C}^{n}\right)$ is injective.

Proof. The Peterson Schubert classes $\left\{\left[X_{w_{J}}^{\prime}\right]\right\}$ form a basis for $H_{*}(\mathfrak{P e t} ; \mathbb{Z})$. Theorem 17 says that the push-forward of each Peterson Schubert class $i_{*}\left(\left[X_{w_{J}}^{\prime}\right]\right)$ has $a_{v(J)}=1$ in its expansion in terms of the Schubert classes, while every other Peterson Schubert class $i_{*}\left(\left[X_{w_{J^{\prime}}}^{\prime}\right]\right)$ has $a_{v(J)}=0$. Thus the Peterson Schubert classes $\left\{i_{*}\left(\left[X_{w_{J}}^{\prime}\right]\right)\right\}$ form a linearly independent set when written in terms of Schubert classes in $H_{*}\left(\operatorname{Flags}\left(\mathbb{C}^{n}\right) ; \mathbb{Z}\right)$. Thus the push-forward $i_{*}: H_{*}(\mathfrak{P e t}, \mathbb{Z}) \rightarrow H_{*}\left(\right.$ Flags $\left.\left(\mathbb{C}^{n}\right), \mathbb{Z}\right)$ is an injection. 


\section{$5 \quad$ An example and open questions}

We conclude with a table giving the Peterson Schubert classes for the Peterson variety in Flags $\left(\mathbb{C}^{4}\right)$. The eight Peterson Schubert subvarieties in $\mathfrak{P e t}_{4}$ correspond to the eight subgroups of $S_{4}$ that are generated by the elements of the power set of $\left\{s_{1}, s_{2}, s_{3}\right\}$. Table 1 shows these Peterson Schubert classes partially expanded in the basis of Schubert classes. The techniques introduced in this paper successfully determine the expansion of each Peterson Schubert class of dimension less than four. We have written

$$
\left[X_{s_{3} s_{2} s_{3} s_{1} s_{2} s_{3}}^{\prime}\right]=\left[X_{s_{1} s_{2} s_{3}}\right]+\left[X_{s_{3} s_{2} s_{1}}\right]+\cdots
$$

because for Peterson Schubert classes of dimension at least four there may be Schubert classes appearing in the expansion that are neither $\left[X_{v(J)}\right]$ nor $\left[X_{u(J)}\right]$.

\begin{tabular}{|l|l|l|l|}
\hline Dimension 3 & Dimension 2 & Dimension 1 & Dimension 0 \\
\hline$\left[X_{s_{3} s_{2} s_{3} s_{1} s_{2} s_{3}}^{\prime}\right]=\left[X_{s_{1} s_{2} s_{3}}\right]+\left[X_{s_{3} s_{2} s_{1}}\right]+\cdots$ & {$\left[X_{s_{1} s_{2} s_{1}}^{\prime}\right]=\left[X_{s_{1} s_{2}}\right]+\left[X_{s_{2} s_{1}}\right]$} & {$\left[X_{s_{1}}^{\prime}\right]=\left[X_{s_{1}}\right]$} & {$\left[X_{e}^{\prime}\right]=\left[X_{e}\right]$} \\
\hline & {$\left[X_{s_{3} s_{2} s_{3}}^{\prime}\right]=\left[X_{s_{2} s_{3}}\right]+\left[X_{s_{3} s_{2}}\right]$} & {$\left[X_{s_{2}}^{\prime}\right]=\left[X_{s_{2}}\right]$} & \\
\hline & {$\left[X_{s_{1} s_{3}}^{\prime}\right]=\left[X_{s_{1} s_{3}}\right]$} & {$\left[X_{s_{3}}^{\prime}\right]=\left[X_{s_{3}}\right]$} & \\
\hline
\end{tabular}

Table 1: Partial expansions of Peterson Schubert classes in terms of Schubert classes

We note that every simple transposition in $J$ appears exactly once in $v(J)$ and $u(J)$. Such permutations are known as Coxeter elements in the group $J \subset S_{n}$. The following are three open questions concerning Coxeter elements in $S_{n}$.

Q1 Can one find a pattern avoidance criterion classifying which Coxeter elements $w(J)$, other than $v(J)$ and $u(J)$, correspond to opposite Schubert varieties $X^{w(J)}$ in the flag variety Flags $\left(\mathbb{C}^{n}\right)$ that are smooth at the point $w_{J}\left(E_{\bullet}\right)$ ? Kumar's Criterion [11, Theorem 5.5] can be used to check which Coxeter elements $w(J)$ correspond to opposite Schubert varieties $X^{w(J)}$ in $\operatorname{Flags}\left(\mathbb{C}^{n}\right)$ that are smooth at $w_{J}\left(E_{\bullet}\right)$ in any Lie type, but since we are restricting our attention to Coxeter elements in Type $A$, it seems plausible that one may find a classification of such Coxeter elements using the combinatorial notion of pattern avoidance similar to the celebrated LakshmibaiSandhya theorem [12].

Q2 If the variety $X^{w(J)}$ is smooth, is the intersection $X_{w_{J}}^{\prime} \cap X^{w(J)}$ proper and transverse?

Q3 For Coxeter elements $w(J)$ other than $v(J)$ and $u(J)$, what are the coefficients $a_{w(J)}$ in the expansion of $\left[X_{w_{J}}^{\prime}\right]=\sum_{a_{u}}\left[X_{u}\right]$ ? 
Question 1 is motivated by the fact that the variety $X^{s_{2} s_{1} s_{4} s_{3}}=X^{31524}$ is singular at $w_{0}\left(E_{\bullet}\right)$, as can be verified using Kumar's Criterion [11, Theorem 5.5 ]. So we know that not every Coxeter element in $S_{n}$ is smooth. Answering Question 2 will be one step toward answering Question 3, since any variety $X^{w(J)}$ satisfying the hypotheses of Question 2 will have $a_{w(J)}=1$.

\section{Acknowledgements}

We would like to thank Megumi Harada, Nicholas Teff, Christie Mauretour, and Julianna Tymoczko for many helpful conversations during the prepation of this paper.

\section{References}

[1] S. Billey and V. Lakshmibai, Singular loci of Schubert varieties, Progr. Math. 182, Birkhauser, Boston, 2000.

[2] A. Bjorner and F. Brenti, Combinatorics of Coxeter Groups. Springer, Berlin, 2003.

[3] M. Brion and J. Carrell, The equivariant cohomology ring of regular varieties, Mich. Math. J. 52, 189-203, 2004

[4] W. Fulton, Intersection Theory. Second Edition, Springer-Verlag, 1998.

[5] M. Goresky and R. MacPherson. On the spectrum of the equivariant cohomology ring. Canad. J. Math., 62, no. 2, 262-283, 2010.

[6] W. Fulton, Young tableaux. With applications to representation theory and geometry. London Math. Soc. Student Texts 35, Cambridge UP, Cambridge, 1997.

[7] M. Harada and J. Tymoczko, A positive Monk formula in the $S^{1}$-equivariant cohomology of type A Peterson varieties. Proc. London Math. Soc. 103, no. 1, 40-72, 2011.

[8] J. Humphreys, Reflection Groups and Coxeter Groups, Cambridge University Press, 1992.

[9] E. Insko and J. Tymoczko, Affine pavings of regular nilpotent Hessenberg varieties and intersection theory of the Peterson variety. arXiv:1309.0484

[10] B. Kostant, Flag Manifold Quantum Cohomology, the Toda Lattice, and the Representation with Highest Weight $\rho$, Selecta Math. (N. S.) no. 2, 43-91, 1996.

[11] S. Kumar. The nil Hecke ring and singularity of Schubert varieties. Invent. Math., 123, no. 3, 471-506, 1996.

[12] V. Lakshmibai and B. Sandhya, Criterion for smoothness of Schubert varieties in SL $(n) / B$. Proc. Indian Acad. Sci. Math. Sci. 100, no. 1, 45-52, 1990.

[13] D. Peterson, Quantum cohomology of $G / P$, Lecture Course, M. I. T., Spring Term 1997.

[14] K. Reitsch, Totally positive Toeplitz matrices and quantum cohomology of partial flag varieties, J. Amer. Math. Soc., 16, no. 2, 363-392, 2003. 
[15] K. Reitsch, Quantum cohomology rings of Grassmannians and total positivity, Duke Math. J., 110, no. 3, 523-553, 2001.

[16] J. Tymoczko, Linear conditions imposed on flag varieties, Amer. J. Math. 128, 1587-1604, 2006.

[17] J. Tymoczko, Paving Hessenbergs by affines, Selecta Math. (N. S.), 13, 353-367, 2007.

[18] A. Woo and A. Yong, Governing singularities of Schubert varieties, J. Algebra, 320, no. 2, 495-520, 2008. 